\title{
Association of Zinc deficiency, oxidative stress and increased double-stranded DNA breaks in globozoospermic infertile patients and its implication for the assisted reproductive technique
}

\author{
Lin Huang ${ }^{1,2,3 \#}$, Guanping Yao ${ }^{4 \#}$, Gelin Huang ${ }^{1,2}$, Chuan Jiang ${ }^{1,2}, \mathrm{Li} \mathrm{Li}^{5}, \mathrm{Lu} \mathrm{Liao}^{5}$, Guiping Yuan ${ }^{6}$, \\ Lijun Shang ${ }^{7}$, Wenming $\mathrm{Xu}^{1,2} \wedge$
}

${ }^{1}$ Joint Laboratory of Reproductive Medicine, SCU-CUHK, Key Laboratory of Obstetric, Gynaecologic and Paediatric Diseases and Birth Defects of Ministry of Education, West China Second University Hospital, Sichuan University, Chengdu, China; ${ }^{2}$ Reproductive Endocrinology and Regulation Laboratory, West China Second University Hospital, Sichuan University, Chengdu, China; ${ }^{3}$ College of Life Science and Technology, Southwest Minzu University, Chengdu, China; ${ }^{4}$ Department of Reproductive Medicine Center, the Affiliated Hospital of Zunyi Medical University, Zunyi, China; ${ }^{5}$ Chengdu PUHUA Technology Co., Ltd., Chengdu, China; ${ }^{6}$ Analytical \& Testing Center, Sichuan University, Chengdu, China; ${ }^{7}$ School of Human Sciences, London Metropolitan University, London, UK

Contributions: (I) Conception and design: L Huang, G Yao, L Shang, W Xu; (II) Administrative support: G Huang, C Jiang, L Li; (III) Provision of study materials or patients: G Yao, L Liao, G Yuan; (IV) Collection and assembly of data: L Huang, L Li, L Liao; (V) Data analysis and interpretation: L Huang, G Yao, L Shang, W Xu; (VI) Manuscript writing: All authors; (VII) Final approval of manuscript: All authors.

\#These authors contributed equally to the work.

Correspondence to: Prof. Wenming Xu. West China Second University Hospital, Sichuan University, No.17, section 3, Renmin South Road, Chengdu 610041, China. Email: xuwenming@scu.edu.cn; Prof. Lijun Shang. School of Human Sciences, London Metropolitan University, 166-220 Holloway Road, London, N7 8DB, UK. Email: 1.shang@londonmet.ac.uk.

Background: Sperm DNA fragmentation and its adverse impact on outcomes of assisted reproductive techniques (ART) in globozoospermic infertile patients has been previously reported. However, the association of Zinc element with DNA damage and intracytoplasmic sperm injection (ICSI) outcome in globozoospermic infertile patients remains unclear.

Methods: Using flame atomic absorption spectrophotometer and superoxide dismutase (SOD) assay, the levels of $\mathrm{Cu}, \mathrm{Fe}, \mathrm{Mn}, \mathrm{Zn}$ and SOD activities in seminal plasma from both globozoospermic infertile patients and fertile volunteers were tested respectively. Using sperm chromatin dispersion (SCD) test and Comet assay, the DNA damages in their semen samples from the two groups was detected. In addition, using Aniline Blue staining, their sperm nucleus maturations were also examined.

Results: The levels of seminal Zinc and SOD activities were lower in the globozoospermic infertile patients and the double-stranded break DFI (DSB-DFI) were significantly higher than that in the fertile controls. Antioxidative insufficiency of SOD with a low $\mathrm{Zn}$ level might be responsible for oxidative stress, which may lead to DNA damage in globozoospermic spermatozoa. Zn deficiency might also have influence on the chromatin stabilization of globozoospermic spermatozoa during spermiogenesis, causing its more vulnerable to oxidative attack.

Conclusions: Serious DSBs in globozoospermia and antioxidative insufficiency due to Zinc element deficiency in spermatozoa might be responsible for the failure of ICSI in globozoospermia.

Keywords: Globozoospermia; Zinc; oxidative stress; sperm DNA damage; intracytoplasmic sperm injection (ICSI)

^ ORCID: Lijun Shang, 0000-0001-5925-5903; Wenming Xu, 0000-0002-3686-229X. 
Submitted Jul 25, 2020. Accepted for publication Dec 28, 2020.

doi: $10.21037 /$ tau-20-1116

View this article at: http://dx.doi.org/10.21037/tau-20-1116

\section{Introduction}

Globozoospermia, primarily characterized by the presence of round-headed spermatozoa with no acrosome, is a severe form of teratozoospermia in male infertility. As a result of deficiency in acrosome, globozoospermic sperm cannot bind and penetrate zona pellucida, resulting in failure of fertilizing an oocyte and then cause sterility $(1,2)$. Failure on in vitro fertilizations were also thought to be related to increased DNA damage in globozoospermic spermatozoa $(3,4)$. Although successful pregnancies have been achieved with globozoospermic spermatozoa using intracytoplasmic sperm injection (ICSI) along with artificial oocyte activation (AOA) (5-7), uncertainty of clinical outcome remains for most cases. Several studies have demonstrated sperm DNA fragmentation and its adverse impact on outcomes of assisted reproductive techniques (ART) in individuals affected by globozoospermia $(4,8,9)$. Therefore, it is imperative to further investigate and monitor the sperm DNA damage levels in these patients.

Sperm DNA damage could be affected by several factors and reactive oxygen species (ROS) production remains one of the most important factors to determine the DNA damage levels in fertilizing sperm $(10,11)$. Sperm are particularly sensitive to ROS due to high proportion of polyunsaturated fatty acids in their membranes, limited ability to repair DNA and intrinsic deficiencies in intracellular antioxidant enzymes $(12,13)$. Fortunately, rich antioxidants in natural seminal plasma can scavenge excess ROS to protect spermatozoa from oxidative injuries, especially after leaving the testis (14). Among these antioxidants, Zinc plays significant roles in the antioxidant networks (15). Zinc ion not only has antioxidative effect for sperm survival and function $(16,17)$ but also can regulate the sperm nuclear chromatin structures $(18,19)$. Moreover, extracellular Zinc ions incorporated into seminal fluid during ejaculation were found to regulate sperm motility (20), sperm morphology (17), membrane stabilization (21) and antioxidant activity for lipid peroxidation (22). In fact, Zinc ion is involved in the whole aspects of sperm structure and function from spermatogenesis, maturation, capacitation, fertilization to embryo development (23). Given the antioxidative roles of Zinc, its deficiency in seminal plasma can lead to oxidative damage induced by ROS. Indeed, Zinc deficiency was reported to result in arrest of spermatogenesis at the round and elongated spermatid stages (24). Reduced level of Zinc ion in seminal plasma was also found in azoospermic, oligozoospermic and asthenozoospermic individuals (25). Apart from Zinc ion, other metal ions such as $\mathrm{Fe}, \mathrm{Mn}$ and $\mathrm{Cu}$ were also reported about their functions as prosthetic groups of SOD activity (26). For example, it was reported that Fe overload likely cause an increase in sperm DNA damage (27) and the level of $\mathrm{Cu}$ and $\mathrm{Mn}$ elements displayed correlation with DNA fragmentation (28). However, whether the level of Zinc ion is associated with sperm DNA damage as well as IVF outcome in globozoospermic infertile patients remain unclear.

Therefore, in this study we investigated the association of Zinc ion dysregulation, oxidative stress, and increased DNA damage and its clinical applications for the globozoospermia patients. Using semen samples from fertile volunteers and globozoospermic individuals who went routine infertile ART, we measured the level of $\mathrm{Zn}, \mathrm{Fe}, \mathrm{Mn}$ and $\mathrm{Cu}$ ions in globozoospermic semen using the flame atomic absorption spectrophotometer. We then tested their SOD activities using the superoxide dismutase (SOD) assay. Their DNA fragmentation level and type of the DNA damage were further investigated through sperm chromatin dispersion (SCD) test, Comet assay, and Aniline Blue staining. We hope this study would help to understand the relationship between serious DSBs and antioxidative insufficiency due to Zinc element deficiency in spermatozoa and provide useful information on future IVF treatment for globozoospermic infertile patients. We present the following article in accordance with the MDAR reporting checklist (available at: http://dx.doi.org/10.21037/tau-20-1116).

\section{Methods}

\section{Subjects and sample collection}

Semen samples were collected from healthy fertile volunteers $(n=12)$ and globozoospermic patients $(n=5)$ who underwent the routine infertile ART outcomes evaluation. The average age of the globozoospermic patients was about $29.2 \pm 4.6$ years. All samples were obtained by masturbation after 2-5 days of sexual abstinence. The seminal samples were collected and evaluated according to $\mathrm{WHO}$ guidelines (29). The study was conducted in accordance with the Declaration of Helsinki 
(as revised in 2013). The study was approved by the Ethical Review Board of West China Second University Hospital, Sichuan University (K2018089). Informed consent was obtained from each subject otherwise the specimens would have been discarded.

\section{Morphologic observation on sperm}

\section{Papanicolaou staining}

Papanicolaou staining were proceeded according to the instruction of the Papanicolaou staining kit (PUHUA, Chengdu, China). Ten $\mu \mathrm{L}$ of semen was smeared onto a glass slide and placed horizontally and air dried. Fixed sufficiently in $95 \%$ alcohol, the coated slides were dipped orderly into the alcohol of $80 \%, 50 \%$ and distilled water. After immersion in the Hematoxylin solution and infiltration with acidic ethanol, the slides were submerged in Orange $\mathrm{G}$ dye following the dehydration in $50 \%, 80 \%$ and $95 \%$ alcohol. When redundant Orange $G$ dyes was removed by continue dissolution in $95 \%$ alcohol, the slides were stained in EA-50 staining solution. Finally, the stained slides underwent dehydration in $95 \%$ and absolute alcohol in turn. At least 200 spermatozoa were scored per sample during morphometric analysis.

\section{Electron microscope observation}

Firstly, semen samples were rinsed in Sperm Washing Medium. Secondly, the semen samples were fixed with $3 \%$ glutaraldehyde, post-fixed by $1 \% \mathrm{OsO}_{4}$ fixative solution, dehydrated in increasing gradient acetone, and then embedded in Epon 812. Before ultrathin sperm-sectioning, a half-thin section was made to ensure that sperm was located under a light microscope. The ultrathin sections were stained with lead citrate-uranyl acetate and then photographed using a transmission electron microscopy (TEM) (TECNAI G2 F20, Philips) under an accelerating voltage of $80 \mathrm{kV}$.

\section{Determination of metal elements and SOD activity in seminal plasma}

\section{Determination of metal elements}

$0.5 \mathrm{~mL}$ of thawed seminal plasma was mixed with $0.5 \mathrm{~mL}$ of $30 \% \mathrm{H}_{2} \mathrm{O}_{2}$. The mixtures were shaken for $30 \mathrm{~min}$, added up to $5 \mathrm{~mL}$ with $5 \% \mathrm{HNO}_{3}$ solution, and mixed thoroughly. Then, the level of $\mathrm{Mn}, \mathrm{Cu}, \mathrm{Zn}$ and Fe elements were determined by using flame atomic absorption spectrophotometer (PerkinElmer PE900T; Waltham,
Massachusetts, USA) according to routine protocol. Certified standards were used as references.

\section{SOD assay}

SOD activity was measured in all samples from fertile volunteers and globozoospermic infertile patients. The seminal plasma was isolated from semen by centrifugation at $2000 \mathrm{rpm}$ for $15 \mathrm{~min}$. SOD activity in the seminal plasma was measured by inhibiting nitrite (NIT) reduction due to the superoxide anion which was generated by the combination of xanthine and xanthine oxidase using a SOD Assay Kit (No. A001-3, Jiancheng bioengineering institute, Nanjing, China). Each of samples were conducted three times as technical replicates. In this reaction system, SOD active unit $(\mathrm{U})$ referred to the enzymatic amount that caused a $50 \%$ inhibition for the rate of NIT reduction, and the SOD activity was definited as $\mathrm{U} / \mathrm{mL}$ seminal plasma.

\section{Detection of DNA fragmentation in semen samples}

\section{SCD test}

An improved SCD test were implemented following the instructions of the SCD kit supplier (PUHUA, China). Briefly, semen samples were diluted to $10 \mathrm{million} / \mathrm{mL}$ in PBS, and $60 \mu \mathrm{L}$ was mixed fully with $120 \mu \mathrm{L}$ of $1 \% \mathrm{LMP}$ agarose (type VII; Sigma, St Louis, USA). Twenty $\mu \mathrm{L}$ of the semen-agarose mixtures were pipetted onto precoated slides and covered with coverslips in the dark until solidification at $4{ }^{\circ} \mathrm{C}$. After removal of the coverslip and incubation in $\mathrm{HCl}$ solution, the slides were submerged in lysing solution and then washed in distilled water. After dehydrated in $50 \%, 70 \%$ and $95 \%$ ethanol, the slides were stained with Wright's solution and washed briefly in tap water and then allowed to dry. Stained sperm cells were visualized using a bright-field microscopy (Olympus, CX31RBSF; Tokyo, Japan) and their image information was collected. Digital images were analyzed using a routine designed with Visilog 7.0 software (Noesis, France). The frequency of sperm cells with fragmented nuclear DNA, i.e., the DNA fragmentation index (DFI), was calculated by counting at least 200 spermatozoa per slide.

\section{Comet assay}

Comet assay test was performed according to a previous protocol (30). Same as in the SCD test, after the gel solidified, coverslips were removed and the slides were submerged in lysing solution 1 for $60 \mathrm{~min}$, followed by lysing solution 2 for $60 \mathrm{~min}$, then washed in TBE buffer. 
For the neutral Comet assay, slides were subjected to electrophoresis in freshly-made $1 \times \mathrm{TBE}$ electrophoresis buffer (pH8.5) at $20 \mathrm{~V}(1 \mathrm{~V} / \mathrm{cm})$ for $12.5 \mathrm{~min}$; For the alkaline Comet assay, slides were incubated in an alkaline denaturing solution for $2.5 \mathrm{~min}$, and then electrophoresed in $0.03 \mathrm{M} \mathrm{NaOH}$ at $20 \mathrm{~V}(1 \mathrm{~V} / \mathrm{cm})$ for $4 \mathrm{~min}$. Both neutral and alkaline slides were rinsed once in the neutralizing solution, thereafter, washed briefly in TBE buffer. Slides finally underwent dehydration in gradient ethanol and air dried. Nucleoid DNA in Comets was stained with SYBR Green I (Gibco, S7585; Grand Island, NY, USA) and visualized fluorescent images were obtained using fluorescence microscope (Nikon 80I, Japan), and stored as TIFF files format with Spot imaging software (Version4.6, Diagnostic instruments Inc., USA). Comet images were analyzed using the CASP software and the thresholds including Head center threshold, Comet threshold, Tail threshold and Head threshold were set as 0.8, 0.05, 0.04 and 0.05 , respectively. At least 400 spermatozoa comets from different fields of view on several slides were counted for each sample. The parameter of the tail moment was chosen as the standard of assessment DNA damage and the value of 0.1 in the tail moment was set as the threshold of sperm with DNA damage in our study. Such threshold values were set by analyzing the mean, median and range of the tail moment values for each assay, and consistent with real ART outcome in the globozoospermic infertile patients and fertile volunteers.

\section{Aniline Blue staining of nuclear maturity of sperm}

Aniline Blue staining was carried out according to the instructions on Aniline Blue Staining Kit (PUHUA, Chengdu, China). After sperm was washed with $0.9 \% \mathrm{NaCl}$, $5 \mu \mathrm{L}$ of the washed samples were spread onto slides and allowed to dry in the air. The smears were fixed in Fast Green solution for $5 \mathrm{~min}$ and subsequently stained with Aniline Blue mixture with acetic acid. After redundant Aniline Blue was removed, the slides were submerged into acid ethanol followed by redyeing with Eosin solution. At least 200 sperm per slide were evaluated and the percentage of sperm with dark blue/blue-purple head was calculated as percentage of immature sperm in nucleus. Stained spermatozoa were evaluated using light microscopy with oil immersion.

\section{Statistical analysis}

Statistical analyses were performed using the Statistics
Package for the Social Sciences software (SPSS, version 26; IBM Crop, Armonk, NY, USA). Ion content, SOD activity and DFI values in SCD and Comet assay as well as sperm maturation were respectively compared between infertile and normal groups using a non-parametric MannWhitney U-test. Unless proposed separately all values were given as mean \pm standard deviation. Values of $\mathrm{P}<0.05$ were considered statistically significant and $\mathrm{P}<0.01$ as extremely significant. The relationships between SOD activity and $\mathrm{Zn}$ level in seminal plasma were examined by Pearson correlation analysis.

\section{Results}

Clinic characteristics and routine infertile ART outcome evaluations of globozoospermic infertile patients

The clinical data of patients were presented in Table 1. Among 5 patients, only Patient No. 2 had one good embryo transferred and achieved clinical pregnancy. Patient No. 3 was Type I globozoospermic patient and had one embryo transferred but failed clinical pregnancy. All other patients didn't receive IVF/ICSI treatment due to personal economic reasons. Their sperm concentration and motility parameters were variable, indicating spermatogenesis and sperm motility were both affected. All the controls were fertile and presented normal sperm parameters according to WHO criteria (29).

\section{Morphological observation on sperm}

Morphologic analysis of semen samples was carried out using Papanicolaou staining to verify the type of globozoospermic sperm. Based on WHO criteria, the stain results showed round-headed and irregular features in sperm of the infertile patients under light microscopy (Figure 1). Further results from electron microscopic clearly displayed the major characteristics of typical globozoospermia, such as round sperm head, the lack of an acrosome and residual cytoplasmic droplets surrounding the nucleus or the midpiece. Also, the chromatin rarefactions distribution was found inside of the nucleus (Figure 2).

\section{Determination of metal elements and SOD activity}

The levels of $\mathrm{Cu}, \mathrm{Fe}, \mathrm{Mn}$ and $\mathrm{Zn}$ ion in seminal plasma from both the fertile volunteers (the control group) and the globozoospermic infertile patients (the globozoospermic group) were tested using flame atomic absorption 
Table 1 The related information on clinical outcomes of the globozoospermic patients involved in the study



ICSI, intracytoplasmic sperm injection.
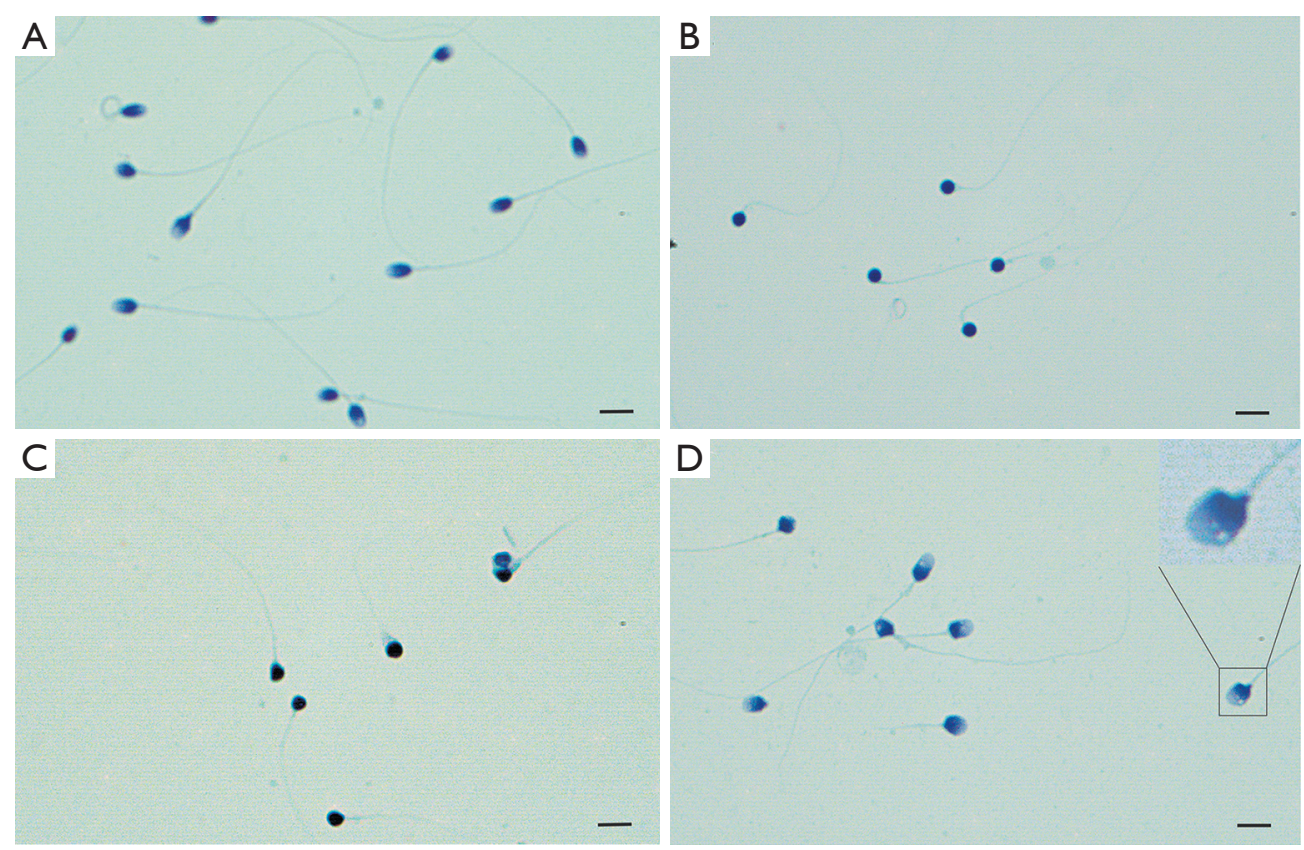

Figure 1 Papanicolaou stain showing morphologic features of globozoospermia (1,000×). (A) Normal sperm head. (B) 100\% round head of type I globozoospermia. (C) Irregular (round, tapered and pyriform) head in type II globozoospermia. (D) Vacuolation in head of globozoospermia (scale bar, $5 \mu \mathrm{m})$.

spectrophotometer. Our results showed that the level of $\mathrm{Zn}$ in seminal plasma from the globozoospermic group was significantly lower than that from the control group $(106.667 \pm 36.147$ vs. $158.274 \pm 31.398 \mu \mathrm{g} / \mathrm{mL}, \mathrm{P}<0.05)$. The level of $\mathrm{Cu}, \mathrm{Fe}, \mathrm{Mn}$ in semen between the globozoospermia and the fertile groups were also tested. There were no significant differences shown (Table 2).

To understand the ability to counteract ROS for the sperm, the SOD activity in seminal plasma was tested in these two groups with different fertility. A lower activity in 

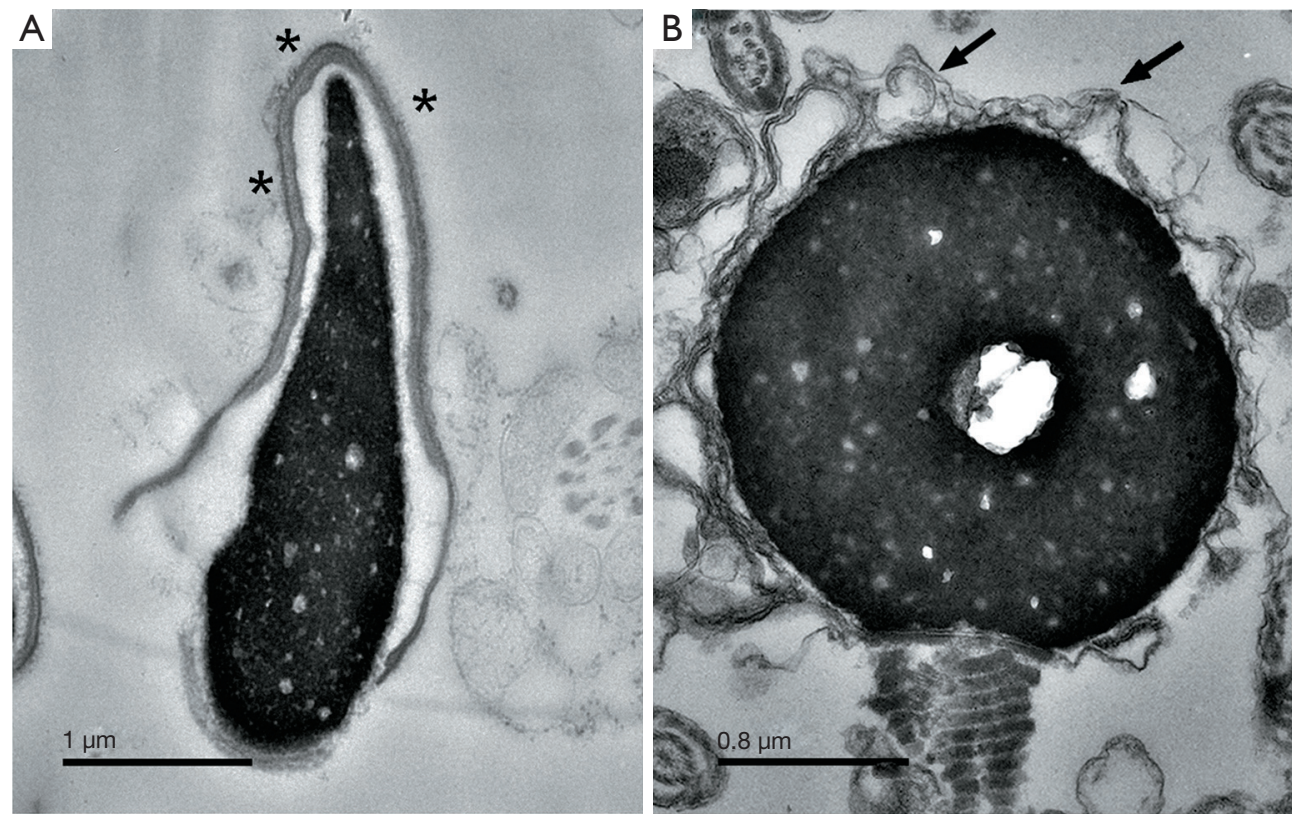

Figure 2 Electron microscopy of globozoospermic sperm. (A) Normal sperm head with acrosome (asterisks). Its elongated shape with a normal length to transversal diameter ratio. (B) A rounded acrosomeless head of globozoospermic sperm. The presence of chromatin rarefaction in the middle of the nucleus and residual cytoplasmic droplets surrounded the round nucleus (black arrows). Panel magnification is indicated by length of the bars.

the globozoospermic group $(319.515 \pm 38.219)$ was observed than that in the control group $(358.148 \pm 23.935)(\mathrm{P}<0.05)$ (Table 2). Statistical analyses displayed that the SOD activity was positively correlated with $\mathrm{Zn}$ content in all semen samples ( $\mathrm{r}=0.485, \mathrm{P}=0.049)$ (Figure 3). Therefore, the above results suggested that in the globozoospermic group, the lower $\mathrm{Zn}$ level was related to the lower SOD activity.

\section{DNA fragmentation analysis}

The SCD test was used to analyze the DNA fragmentation produced in the sperm. The nuclear chromatin was very well preserved with a high density whereby the halos can be accurately stained and assessed by bright-field microscopy (Figure 4). The mean DFI revealed by SCD test was significantly higher in the globozoospermic group compared with that in the control group $(58.30 \% \pm 4.63 \%$ vs. $23.44 \% \pm 4.46 \%)(\mathrm{P}<0.01)$ (Table $\mathrm{S} 1)$. This result indicated there was significant amount of DNA fragmentation produced in the globozoospermic spermatozoa according to the WHO criteria (DFI value $>30 \%$ ).

Subsequently, the Comet assay was carried out since the assay can not only identify the total level of sperm
DNA fragmentation but also distinguish different types of DNA breaks of single-stranded DNA break (SSB) and double-stranded DNA break (DSB). This allowed a more precise and extensive analysis of the DNA damage. To assess the level of DNA fragmentation in sperm, a threshold for quantitative parameters such as tail length, olive tail moment is normally established according to previous studies $(31,32)$, or the mean of DNA breaks in ART outcome is usually calculated as threshold values of Comet assay results $(33,34)$. Our results showed that a significant higher double-stranded break DFI (DSB-DFI) in the globozoospermia than that in the control group (38.36 \pm 11.88 vs. $14.09 \pm 1.95, \mathrm{P}<0.01)$. As shown in Table 3 , the DSB-DFI $(38.36 \pm 11.88)$ was also higher than SSBDFI $(18.59 \pm 6.32)$ in the globozoospermic group. Moreover, the levels of the section with low SDF within DSB were also dramatically higher in globozoospermic group than in the control group $(23.36 \pm 10.24$ vs. $7.91 \pm 1.94)(\mathrm{P}<0.01)$. This meant that the incidence of sperm with short comet tail was relatively high in the globozoospermic sample. There was no difference in total SSB-DFI between the globozoospermic group and the control group whereas the proportion of high levels of SDF within total SSB-DFI 
Table 2 Four elements content and SOD activity in seminal plasm from globozoospermic patients and fertile controls

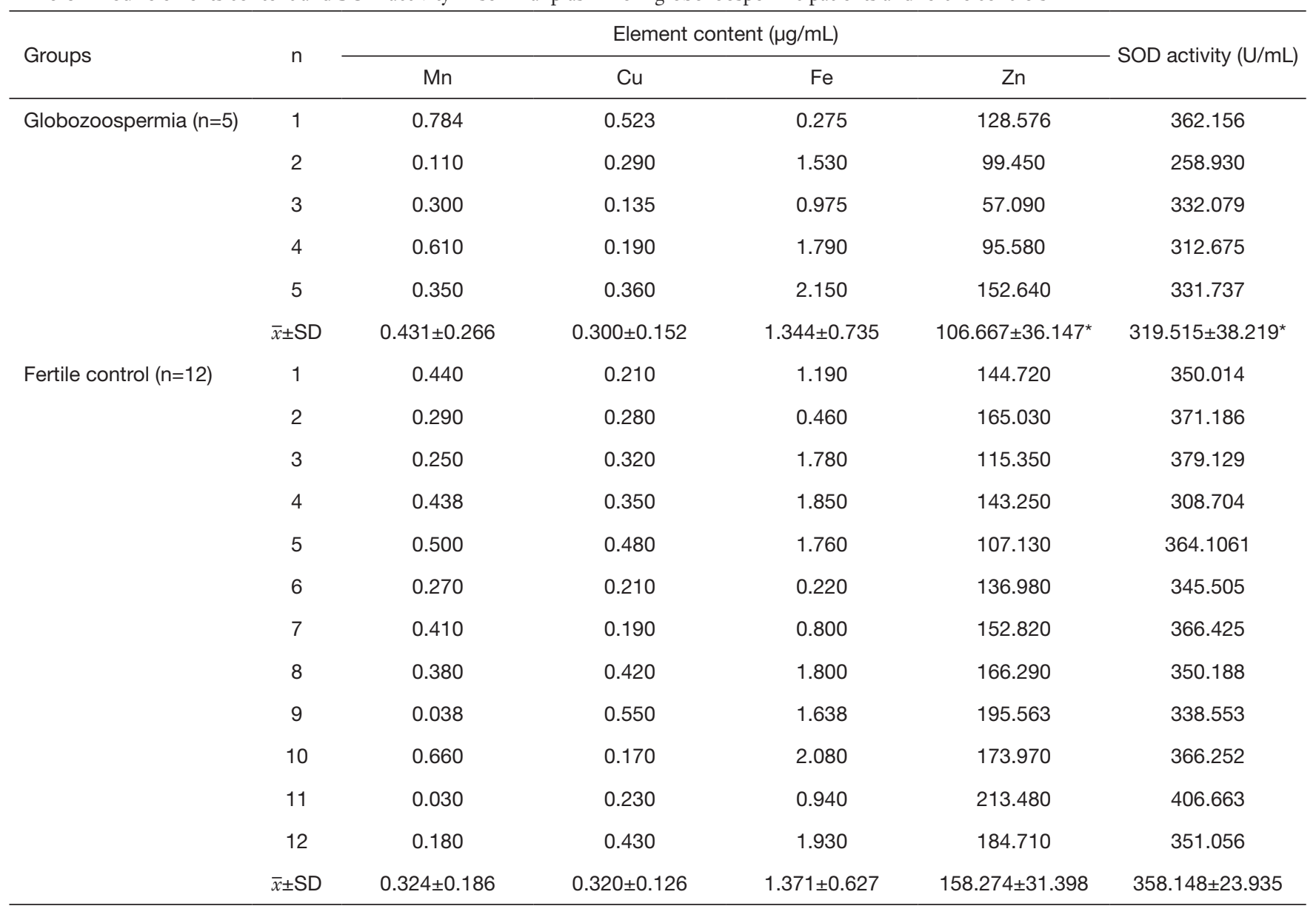

${ }^{*}$, significant differences, with respect to fertile controls $(\mathrm{P}<0.05)$. SOD, superoxide dismutase.

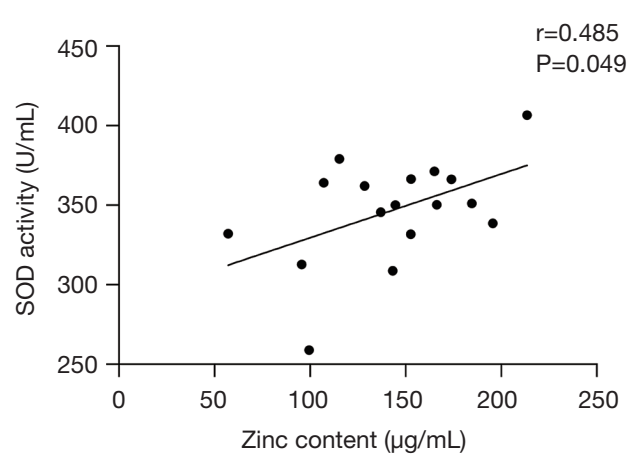

Figure 3 Correlation analysis between SOD activity and Zinc concentration in seminal plasma. SOD, superoxide dismutase.

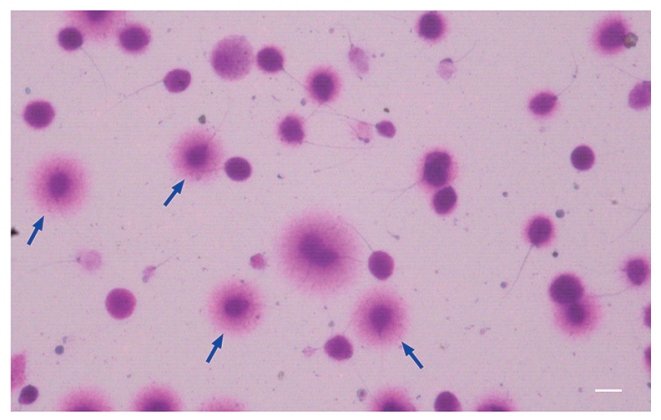

Figure 4 Representative Sperm Chromatin Dispersion (SCD) test on one globozoospermic patient $(400 \times)$. Sperm cells with halo (blue arrows) showed normal sperm chromatin dispersion. The frequency of sperm cells without halo was reflective of a degree of nuclear damage (scale bar, $10 \mu \mathrm{m}$ ). 
Table 3 DSB-DFI and SSB-DFI calculated from semen samples from infertile patients and fertile control subjects

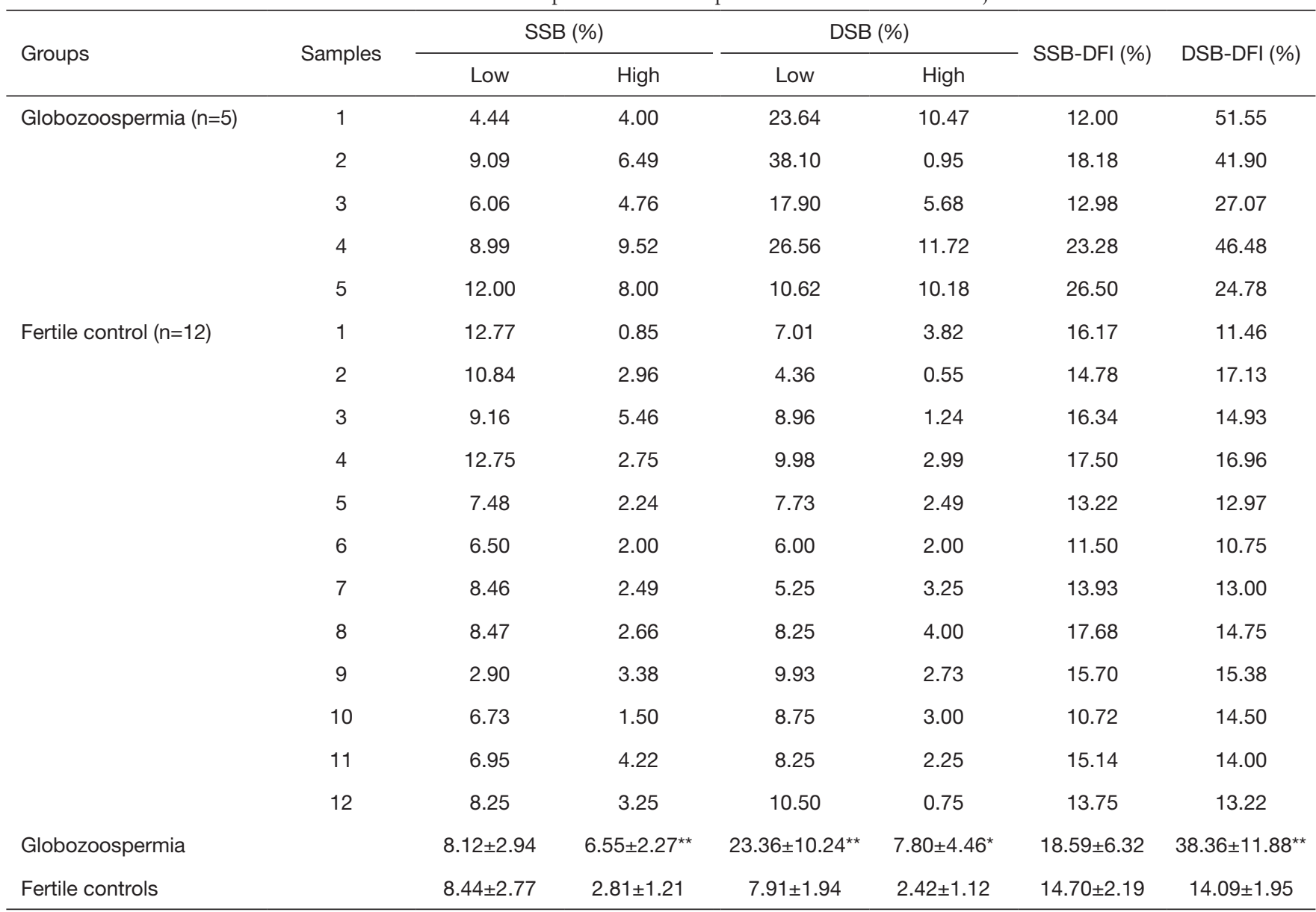

*, $\mathrm{P}<0.05$, ${ }^{* *}, \mathrm{P}<0.01$, respectively, globozoospermia compared with fertile control groups. DFI, frequency of the sperm DNA fragmentation (SDF); SSB-DFI, frequency of single-stranded breaks; DSB-DFI, frequency of double-stranded breaks. The percentage of high and low levels of SDF was respectively counted within SSB and DSB for each sample according to the threshold of tail moment in comet results. The tail moment values of $0.1-0.5$ and $>1.5$ respectively were set as low and high SSB while $0.1-10$ and $>25$ values were set for low and high DSB, respectively.

between the two groups was significantly different $(\mathrm{P}<0.01)$ (Table 3), indicating the serious extent rather than universality of single strand fragment in globozoospermic infertile patients.

\section{Nuclear maturation of sperm}

During spermiogenesis, the replacement of histones by protamines allows spermatozoa to acquire a highly packed mature structure. Aniline blue selectively binding to lysinerich histones while Eosin specifically combing with rich arginine and cystine residues of protamines in spermatozoa showed the presence of histones for blue and protamines for red in the sperm nucleus respectively (Figure 5). Thus, the indirect approach was used to evaluate mature status of sperm by calculating the ratio of the sperm with extra histones in the sperm nucleus for sperm samples. Our results showed the percentage of reacted spermatozoa to Aniline Blue in the globozoospermic group $(64.51 \% \pm 15.31 \%)$ was significant higher (up to 2-fold) than that in the control group $(29.67 \% \pm 9.97 \%)(\mathrm{P}<0.01)$ (Figure $5 D)$. This meant that histones which should have been successfully replaced by protamines in sperm nucleus were excessive present, in other words, there was more abnormal immature sperm in globozoospermic infertile patients.

\section{Discussion}

Understanding the DNA damage in patients with 

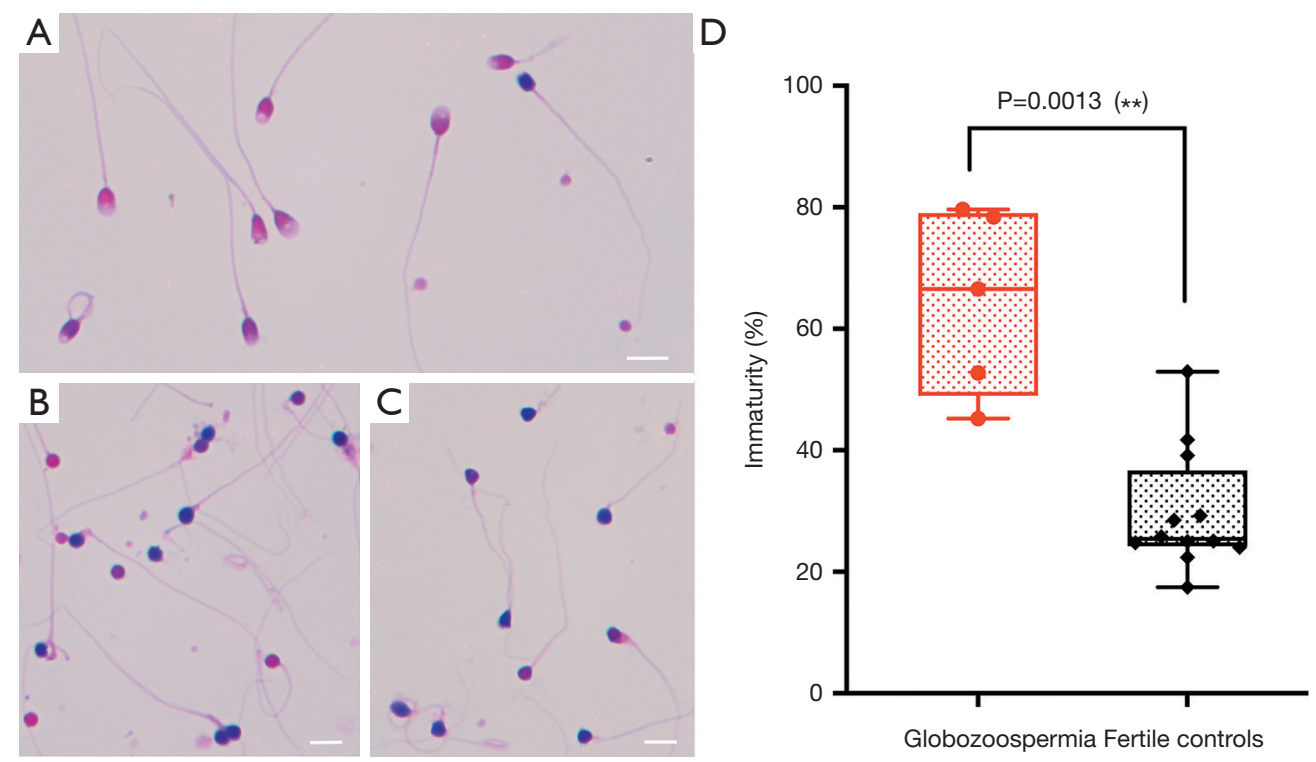

Figure 5 Aniline blue staining $(1,000 \times)$. Sperm with normal protamine (red stained) and immature sperm with low protamine or high histone (while dark blue/blue-purple stained). (A) Normal sperm. (B) Sperm from globozoospermia I. (C) Sperm from globozoospermia II. (D) Box-plot of nuclear immaturity between globozoospermia and fertile control groups, ${ }^{* *}, \mathrm{P}<0.01$ (scale bar, $5 \mu \mathrm{m}$ ).

teratozoospermia and providing intervention to improve the quality of sperm is crucial to the success of conception in ART (35-39), In this study, we used both SCD test and Comet assay to exam globozoospermia samples and observed obvious DNA fragmentation and severe DSBs. A significant increase of DSB in sperm might explain the failure of ICSI in these patients compared with the clinical evaluation data of patients on ART (Table 1). We also found that the level of seminal Zinc and SOD activities were lower in the globozoospermic infertile patients and their DSB-DFI was significantly higher than that in the fertile controls. Anti-oxidative insufficiency of SOD with low $\mathrm{Zn}$ level therefore might be responsible for oxidative stress, which may lead to higher DNA damage in globozoospermic spermatozoa.

To our knowledge, this is the first study which systemically investigate the ion concentration, oxidative stress marker and DFI index in globozoospermia samples. A correlation of low $\mathrm{Zn}$ level with higher DFI index observed in this study indicated that a low $\mathrm{Zn}$ level may be responsible for the high oxidative stress and the higher DNA damage in sperm. However, this needs to be further confirmed with a larger cohort of samples as this study only included 5 samples due to its rare availability. Using simple Comet assay, this study enabled the identification of DNA fragmentation and clear characterisation in the sperm from teratozoospermic infertile patients. Identifying the association of $\mathrm{Zn}$, SOD activities and DNA fragmentation in spermatozoa patients showed potentially how the reduced SOD activities under zinc deficiency could disturb the balance and be responsible for oxidative stress in globozoospermia. Understanding this important correlation of zinc and globozoospermia could provide a useful treatment strategy for teratozoospermic infertile patients in their ART treatment.

\section{DNA fragmentations in spermatozoa patients and its clinical implications}

Men with teratozoospermia would normally need ART to achieve conception, but the outcome is often very poor at the present and many patients are even confronted with a higher risk of selecting spermatozoa with damaged DNA for ART $(35,36)$. Overwhelming evidences suggest that spermatozoa with DNA fragmentation is associated with higher miscarriage rates, lower good-quality embryo rate, lower clinical pregnancy rate and lower live birth rate after ART (37-39). In this study, using Comet assay we were able to characterise the DNA fragmentation and compare the parameters related to DSB/SSB in spermatozoa patients to determine how the different types of DSB could have impact on the IVF/ICSI outcome. 
There are normally two types of DSB, extensive DSB and localized DSB. The extensive DSB has very long comet tails separated from the sperm core and is very small part of total DSB, while the localized DSB is the most common DSB and largely localized and attached to the matrix attachment regions (MAR) (40). One previous study reported that a large number of sperm with extensive DSB (long tails) and those with SSB were present in a normal seminal sample (40). On the contrary, our data showed the higher incidence of sperm with DSB (short Comet tail) as well as relatively lower SSB-DFI in the globozoospermic samples than the normal controls. Therefore, it seemed obvious that localized DSB in the MAR was the main form of DSB in our globozoospermic sperms. The report has also claimed that localized DSB in the MAR of human sperm may lead to the risks of recurrent miscarriage and implantation failure as well as slow embryo kinetics in ICSI cycles (40). In our study, there were also significant differences in the proportion of high fragmentation levels within total SSB-DFI between the globozoospermic patients and the fertile controls, suggesting the presence of high level SSB and poor ability to repair SSB for globozoospermia. The impaired function of nuclear matrix due to its damaged integrity caused by the localized DSB have been reported to be the possible reason for the failure outcomes of ART $(41,42)$. The localized DSB has also been demonstrated to attach to the sperm nuclear matrix probably through the TOP2B or similar protein $(43,44)$. It is now clear that topoisomerase II mediates DSBs in somatic cells (45). In spermatozoa, supraphysiological ROS levels disturb sperm TOPII, which is involved in the nuclear remodeling via breaking and reconnecting DNA during spermiogenesis, causing large amounts of DNA nicks in elongating spermatids, and finally result in sperm DNA fragmentation (46). Our results showed the higher incidence of sperm with DSB (short Comet tail) as well as relatively lower SSB-DFI could therefore be used to predict the outcome of IVF/ ICSI in globozoospermic spermatozoa, and possibly in other types of teratozoospermia samples. However, this needs to be further validated in larger cohort of samples.

\section{The role of Zn in spermatozoa patients and its clinical implications}

Ion measurement on semen samples in this study showed that $\mathrm{Zn}$ deficiency is one of the important characteristics in globozoospermic spermatozoa samples. In addition to be a cofactor for various proteins and electron transport, $\mathrm{Zn}$ was reported to be crucial in the stabilization of sperm chromatin $(18,47)$. When the condensation of the nucleus starts, Zinc is incorporated into the sperm nucleus and participates in the process of protamination by the formation of S-Zn-S bonds with protamine, which maintains the stabilization of chromatin during spermiogenesis (18). We observed abnormal sperm chromatin in form of impaired chromatin condensations by TEM in the globozoospermic samples (Figure 2B). At the same time, the Aniline Blue staining also displayed protamination level deficiency in sperm nucleus of the infertile patients (Figure 5). This is entirely possible that in our patients with globozoospermia, the impaired chromatin compaction induced DNA breaks and free radicals by reducing protamination and disulphide bond formation owing to deficient $\mathrm{Zn}$ (48). Alternatively, in the absence of Zinc, globozoospermic spermatozoa carrying poor chromatin compaction is more vulnerable to oxidative attack (49). In fact, the $\mathrm{Zn}$ level in the sperm of Patient No. 3 is the lowest and he was a type I globozoospermic patient. This indicated that a low $\mathrm{Zn}$ level might be responsible for his unsuccessful ICSI outcome. Moreover, reduced Zinc transport proteins might be another possible cause for $\mathrm{Zn}$ dysfunction because they regulate Zinc uptake into the cytoplasm or from the lumen of intracellular compartments (50,51). A novel member of RING finger proteins, the RNF151, was found to possess a TRAF-type Zinc finger domain and $\mathrm{C} 3 \mathrm{HC} 4-$ type RING finger domain, and this Zinc finger protein probably plays a role in acrosome formation by interaction with dysbindin (52). All the above suggested that Zinc might function in acrosomal biogenesis indirectly.

Reportedly, oral Zinc supplementation can restore the level of the semen parameters including semen volume, sperm count, progressive sperm motility, normal sperm form in asthenozoospermic subjects. At the same time, the Zinc supplementation also returns to normal value for HMW-Zn, one of three types of Zinc binding proteins, which include high (HMW), intermediate (IMW), and low molecular weight ligand (LMW) proteins in human seminal plasma (53). Clinical results indicated that zinc sulfate treatment for infertile patients with low Zinc level in semen may be an effective therapy for infertile individuals especially after varicocelectomy (54). Our results in this study, together with previous studies, certainly provided more evidences and inspiration to treat globozoospermia by zinc supplementation in the future. 


\section{Association of Zn, SOD activities and DNA fragmentation in spermatozoa patients and its clinical implications}

In this study, we investigated the association of reduced SOD activity and the $\mathrm{Zn}$ level in the sperm samples of spermatozoa patients. SODs initiate the conversion of superoxide anion and scavenge superoxide free radical whereby spermatozoa maintain membrane fluidity and internal environments, thereby ensuring the normal morphologic structures and function for sperm $(55,56)$. It has been found that SOD activity was negatively associated with sperm DNA fragmentation (57). Given the crucial importance of SOD in defense strategy, the level of $\mathrm{Zn}, \mathrm{Cu}$, $\mathrm{Mn}$ and Fe elements in semen and SOD activity in seminal plasma were examined in the globozoospermic and normal samples in this study. Previous studies found a higher level of $\mathrm{Fe}$ content presented in semen from teratozoospermia than that from the spermatozoa patients, and therefore caused morphological anomalies of spermatozoa due to Fe-induced lipid peroxidation (58-60). Unlike these results, our results did not establish the difference between globozoospermia and normal samples in the level of Fe, together with $\mathrm{Cu}$ and $\mathrm{Mn}$ elements. However, we observed a strong positive correlation between SOD activity and the level of $\mathrm{Zn}$ in semen, which confirmed previous reports on the zinc-dependent enzymatic activity of the sperm $(58,61)$.

Testes contain cytosolic (CuZnSOD), mitochondrial (MnSOD) and secretory extracellular SOD (Ex-SOD) forms of SOD (26). Among them, 75\% activities attribute to CuZnSOD, the main SOD isoform in spermatozoa SOD. In this study, the level of $\mathrm{Zn}$ content and SOD activities were both found significantly lower in globozoospermic semen. Thus, we assumed that this compromised SOD activities could be attributed to Zinc deficiency in globozoospermia. It could be the case that the expression of SOD gene actually increased probably as a response to oxidative stress, but their activities still remained at a low level due to the lack of Zinc as a prosthetic group. It has been reported that for proper function of spermatozoa, the adequate balance is vital between superoxide radical generation and dismutation (62). Therefore, it is well accepted that reduced SOD activities under Zinc deficiency would necessarily disturb the balance and be responsible for oxidative stress in globozoospermia. Our results further confirmed this. Understanding this important correlation could provide useful treatment strategies for teratozoospermia patients in their ART treatment.

In conclusion, our study found that serious DSBs were presented in globozoospermia and occurred mainly in MAR of sperm nucleus. Antioxidative insufficiency of SOD under the lack of $\mathrm{Zn}$ might be responsible for oxidative stress, which led to DNA damage in globozoospermic spermatozoa. Zn deficiency might also have influence on the chromatin stabilization of globozoospermic spermatozoa during spermiogenesis, resulting in its more vulnerable to oxidative attack. All the above suggested that it is critical to determine a detailed profile of the $\mathrm{Zn}$ level, oxidative stress marker, SOD and DSB value for globozoospermic infertile patients so that more comprehensive outcome prediction of ICSI can be planned for these patients. To help to achieve a high successful rate of ICSI, we hypothesize that Zinc therapy for patients suffering from globozoospermia can be beneficial in improving ART outcomes.

\section{Acknowledgments}

We are grateful for the participation of all patients and donors in this study. The authors would like to thank to Mr. Zhang Chunjie (Chengdu PUHUA Co., Ltd) and Dr. Xu Qian'nan (West China School of Basic Medical Sciences \& Forensic Medicine, Sichuan University) as well as Dr. Wang Meixian (West China Second University Hospital, Sichuan University) for the technical supports in Papanicolaou staining, Comet assay and statistical analysis, respectively. Funding: This study was supported by National Key Research \& Development program of China (No. 2018YFC1003603), and the National Nature Science Fund of China (No. 81771642, 21572147).

\section{Footnote}

Reporting Checklist: The authors have completed the MDAR reporting checklist. Available at http://dx.doi.org/10.21037/ tau-20-1116

Data Sharing Statement: Available at http://dx.doi. org/10.21037/tau-20-1116

Conflicts of Interest: All authors have completed the ICMJE uniform disclosure form (available at http://dx.doi. org/10.21037/tau-20-1116). The authors have no conflicts of interest to declare.

Ethical Statement: The authors are accountable for all aspects of the work in ensuring that questions related to the accuracy or integrity of any part of the work are 
appropriately investigated and resolved. The study was conducted in accordance with the Declaration of Helsinki (as revised in 2013). The study was approved by the Ethical Review Board of West China Second University Hospital, Sichuan University (K2018089). Informed consent was obtained from each subject otherwise the specimens would have been discarded.

Open Access Statement: This is an Open Access article distributed in accordance with the Creative Commons Attribution-NonCommercial-NoDerivs 4.0 International License (CC BY-NC-ND 4.0), which permits the noncommercial replication and distribution of the article with the strict proviso that no changes or edits are made and the original work is properly cited (including links to both the formal publication through the relevant DOI and the license). See: https://creativecommons.org/licenses/by-nc-nd/4.0/.

\section{References}

1. Dam AH, Feenstra I, Westphal JR, et al. Globozoospermia revisited. Hum Reprod Update 2007;13:63-75.

2. Han F, Liu C, Zhang L, et al. Globozoospermia and lack of acrosome formation in GM130-deficient mice. Cell Death Dis 2017;8:e2532.

3. Talebi AR, Ghasemzadeh J, Khalili MA, et al. Sperm chromatin quality and DNA integrity in partial versus total globozoospermia. Andrologia 2018. doi: 10.1111/ and.12823.

4. Deemeh MR, Tavalee M, Razavi S, et al. Evaluation of Protamine Deficiency and DNA Fragmentation in Two Globozoospermia Patients Undergoing ICSI. Iranian J Fertil Steril 2007;1:85-8.

5. Nasr-Esfahani MH, Deemeh MR, Tavalaee M. Artificial oocyte activation and intracytoplasmic sperm injection. Fertil Steril 2010;94:520-6.

6. Tavalaee M, Nomikos M, Lai FA, et al. Expression of sperm PLC $\zeta$ and clinical outcomes of ICSI-AOA in men affected by globozoospermia due to DPY19L2 deletion. Reprod Biomed Online 2018;36:348-55.

7. Taylor SL, Yoon SY, Morshedi MS, et al. Complete globozoospermia associated with PLCzeta deficiency treated with calcium ionophore and ICSI results in pregnancy. Reprod Biomed Online 2010;20:559-64.

8. Vozdova M, Rybar R, Kloudova S, et al. Total globozoospermia associated with increased frequency of immature spermatozoa with chromatin defects and aneuploidy: a case report. Andrologia 2014;46:831-6.
9. Eskandari N, Tavalaee M, Zohrabi D, et al. Association between total globozoospermia and sperm chromatin defects. Andrologia 2018. doi: 10.1111/and.12843.

10. Aitken RJ. Reactive oxygen species as mediators of sperm capacitation and pathological damage. Mol Reprod Dev 2017;84:1039-52.

11. Homa ST, Vassiliou A, Stone J, et al. A Comparison Between Two Assays for Measuring Seminal Oxidative Stress and their Relationship with Sperm DNA Fragmentation and Semen Parameters. Genes (Basel) 2019;10:236.

12. Lenzi A, Gandini L, Picardo M, et al. Lipoperoxidation damage of spermatozoa polyunsaturated fatty acids (PUFA): scavenger mechanisms and possible scavenger therapies. Front Biosci 2000;5:E1-E15.

13. Talevi R, Barbato V, Fiorentino I, et al. Protective effects of in vitro treatment with zinc, $d$-aspartate and coenzyme q10 on human sperm motility, lipid peroxidation and DNA fragmentation. Reprod Biol Endocrinol 2013;11:81.

14. Aitken RJ, Roman SD. Antioxidant systems and oxidative stress in the testes. Oxid Med Cell Longev 2008;1:15-24.

15. Ganger R, Garla R, Mohanty BP, et al. Protective Effects of Zinc Against Acute Arsenic Toxicity by Regulating Antioxidant Defense System and Cumulative Metallothionein Expression. Biol Trace Elem Res 2016;169:218-29.

16. Macanovic B, Vucetic M, Jankovic A, et al. Correlation between sperm parameters and protein expression of antioxidative defense enzymes in seminal plasma: a pilot study. Dis Markers 2015;2015:436236.

17. Colagar AH, Marzony ET, Chaichi MJ. Zinc levels in seminal plasma are associated with sperm quality in fertile and infertile men. Nutr Res 2009;29:82-8.

18. Björndahl L, Kvist U. Human sperm chromatin stabilization: a proposed model including zinc bridges. Mol Hum Reprod 2010;16:23-9.

19. Kvist U. Importance of spermatozoal zinc as temporary inhibitor of sperm nuclear chromatin decondensation ability in man. Acta physiol Scand 1980;109:79-84 .

20. Riffo M, Leiva S, Astudillo J. Effect of zinc on human sperm motility and the acrosome reaction. Int J Androl 1992;15:229-37.

21. Bettger WJ, O'Dell BL. A critical physiological role of zinc in the structure and function of biomembranes. Life Sci 1981;28:1425-38.

22. Bray TM, Bettger WJ. The physiological role of zinc as an antioxidant. Free Radic Biol Med 1990;8:281-91.

23. Kerns K, Zigo M, Sutovsky P. Zinc: A Necessary Ion for 
Mammalian Sperm Fertilization Competency. Int J Mol Sci 2018;19:4097.

24. Omu AE, Al-Azemi MK, Al-Maghrebi M, et al. Molecular basis for the effects of zinc deficiency on spermatogenesis: An experimental study in the Sprague-dawley rat model. Indian J Urol 2015;31:57-64.

25. Khan MS, Zaman S, Sajjad M, et al. Assessment of the level of trace element zinc in seminal plasma of males and evaluation of its role in male infertility. Int J Appl Basic Med Res 2011;1:93-6.

26. Marklund SL. Extracellular superoxide dismutase and other superoxide dismutase isoenzymes in tissues from nine mammalian species. Biochem J 1984;222:649-55.

27. Perera D, Pizzey A, Campbell A, et al. Sperm DNA damage in potentially fertile homozygous betathalassaemia patients with iron overload. Hum Reprod 2002;17:1820-5.

28. Kovacik A, Tirpak F, Tomka M, et al. Trace elements content in semen and their interactions with sperm quality and RedOx status in freshwater fish Cyprinus carpio: A correlation study. J Trace Elem Med Biol 2018;50:399-407.

29. World Health Organization. WHO laboratory manual for the examination and processing of human semen. Fifth edition. 2010.

30. Enciso M, Sarasa J, Agarwal A, et al. A two-tailed Comet assay for assessing DNA damage in spermatozoa. Reprod Biomed Online 2009;18:609-16.

31. Morris ID, Ilott S, Dixon L, et al. The spectrum of DNA damage in human sperm assessed by single cell gel electrophoresis (Comet assay) and its relationship to fertilization and embryo development. Hum Reprod 2002;17:990-8.

32. Tomsu M, Sharma V, Miller D. Embryo quality and IVF treatment outcomes may correlate with different sperm comet assay parameters. Hum Reprod 2002;17:1856-62.

33. Simon L, Lutton D, McManus J, et al. Sperm DNA damage measured by the alkaline Comet assay as an independent predictor of male infertility and in vitro fertilization success. Fertil Steril 2011;95:652-7.

34. Simon L, Liu L, Murphy K, et al. Comparative analysis of three sperm DNA damage assays and sperm nuclear protein content in couples undergoing assisted reproduction treatment. Hum Reprod 2014;29:904-17.

35. French DB, Sabanegh ES, Goldfarb J, et al. Does severe teratozoospermia affect blastocyst formation, live birth rate, and other clinical outcome parameters in ICSI cycles? Fertil Steril 2010;93:1097-103.

36. Simon L, Murphy K, Shamsi MB, et al. Paternal influence of sperm DNA integrity on early embryonic development. Hum Reprod 2014;29:2402-12.

37. Deng C, Li T, Xie Y, et al. Sperm DNA fragmentation index influences assisted reproductive technology outcome: A systematic review and meta-analysis combined with a retrospective cohort study. Andrologia 2019;51:e13263.

38. Osman A, Alsomait H, Seshadri S, et al. The effect of sperm DNA fragmentation on live birth rate after IVF or ICSI: a systematic review and meta-analysis. Reprod Biomed Online 2015;30:120-7.

39. Borini A, Tarozzi N, Bizzaro D, et al. Sperm DNA fragmentation: paternal effect on early postimplantation embryo development in ART. Hum Reprod 2006;21:2876-81.

40. Ribas-Maynou J, Benet J. Single and Double Strand Sperm DNA Damage: Different Reproductive Effects on Male Fertility. Genes (Basel) 2019;10:105.

41. Shaman JA, Yamauchi Y, Ward WS. The sperm nuclear matrix is required for paternal DNA replication. J Cell Biochem 2007;102:680-8.

42. Shaman JA, Yamauchi Y, Ward WS. Function of the sperm nuclear matrix. Arch Androl 2007;53:135-40.

43. Ribas-Maynou J, Gawecka JE, Benet J, et al. Doublestranded DNA breaks hidden in the neutral Comet assay suggest a role of the sperm nuclear matrix in DNA integrity maintenance. Mol Hum Reprod 2014;20:330-40.

44. Gawecka JE, Ribas-Maynou J, Benet J, et al. A model for the control of DNA integrity by the sperm nuclear matrix. Asian J Androl 2015;17:610-5.

45. Uusküla-Reimand L, Wilson MD. Break Check: Transcription-Driven Topoisomerase II Collisions near Chromatin Loop Anchors Are Hotspots for DNA Damage and Translocations. Mol Cell 2019;75:203-5.

46. Fesahat F, Henkel R, Agarwal A. Globozoospermia syndrome: An update. Andrologia 2020;52:e13459.

47. Kvist U, Kjellberg S, Bjorndahl L, et al. Zinc in sperm chromatin and chromatin stability in fertile men and men in barren unions. Scand J Urol Nephrol 1988;22:1-6.

48. Oumaima A, Tesnim A, Zohra H, et al. Investigation on the origin of sperm morphological defects: oxidative attacks, chromatin immaturity, and DNA fragmentation. Environ Sci Pollut Res Int 2018;25:13775-86.

49. Aitken RJ, De Iuliis GN. On the possible origins of DNA damage in human spermatozoa. Mol Hum Reprod 2010;16:3-13.

50. Croxford TP, McCormick NH, Kelleher SL. Moderate zinc deficiency reduces testicular Zip6 and Zip10 abundance and impairs spermatogenesis in mice. J Nutr 
2011;141:359-65.

51. Chu Q, Chi ZH, Zhang X, et al. A potential role for zinc transporter 7 in testosterone synthesis in mouse Leydig tumor cells. Int J Mol Med 2016;37:1619-26.

52. Nian H, Fan C, Liao S, et al. RNF151, a testis-specific RING finger protein, interacts with dysbindin. Arch Biochem Biophys 2007;465:157-63.

53. Hadwan MH, Almashhedy LA, Alsalman AR. Oral zinc supplementation restores high molecular weight seminal zinc binding protein to normal value in Iraqi infertile men. BMC Urol 2012;12:32.

54. Takihara H, Cosentino MJ, Cockett AT. Zinc sulfate therapy for infertile male with or without varicocelectomy. Urology 1987;29:638-41.

55. Shiva M, Gautam AK, Verma Y, et al. Association between sperm quality, oxidative stress, and seminal antioxidant activity. Clin Biochem 2011;44:319-24.

56. Tavilani H, Goodarzi MT, Vaisi-raygani A, et al. Activity of antioxidant enzymes in seminal plasma and their relationship with lipid peroxidation of spermatozoa. Int

Cite this article as: Huang L, Yao G, Huang G, Jiang C, Li L, Liao L, Yuan G, Shang L, Xu W. Association of Zinc deficiency, oxidative stress and increased double-stranded DNA breaks in globozoospermic infertile patients and its implication for the assisted reproductive technique. Transl Androl Urol 2021;10(3):1088-1101. doi: 10.21037/tau-20-1116
Braz J Urol 2008;34:485-91.

57. Yan L, Liu J, Wu S, et al. Seminal superoxide dismutase activity and its relationship with semen quality and SOD gene polymorphism. J Assist Reprod Genet 2014;31:549-54.

58. Marzec-Wróblewska U, Kaminski P, Lakota P, et al. Zinc and iron concentration and SOD activity in human semen and seminal plasma. Biol Trace Elem Res 2011;143:167-77.

59. Aydemir B, Kiziler AR, Onaran I, et al. Impact of $\mathrm{Cu}$ and Fe concentrations on oxidative damage in male infertility. Biol Trace Elem Res 2006;112:193-203.

60. Ammar O, Houas Z, Mehdi M. The association between iron, calcium, and oxidative stress in seminal plasma and sperm quality. Environ Sci Pollut Res Int 2019;26:14097-105.

61. Yeung CH, Cooper TG, De Geyter M, et al. Studies on the origin of redox enzymes in seminal plasma and their relationship with results of in-vitro fertilization. Mol Hum Reprod 1998;4:835-9.

62. Sikka SC. Relative impact of oxidative stress on male reproductive function. Curr Med Chem 2001;8:851-62. 
Supplementary

Table S1 DFI results in globozoospermia and normal control with the SCD method

\begin{tabular}{|c|c|c|c|c|c|}
\hline Groups & Sample ID & Number without halo & Total count & $\mathrm{DFI}(\%)$ & Mean \pm SD $(\%)$ \\
\hline \multirow[t]{5}{*}{ Globozoospermia $(n=5)$} & 1 & 159 & 252 & 63.09 & \\
\hline & 2 & 121 & 209 & 57.89 & \\
\hline & 3 & 122 & 222 & 54.95 & $58.30 \pm 4.63^{\star *}$ \\
\hline & 4 & 106 & 201 & 52.74 & \\
\hline & 5 & 147 & 234 & 62.82 & \\
\hline \multirow[t]{12}{*}{ Fertile controls $(n=12)$} & 1 & 79 & 337 & 23.44 & \\
\hline & 2 & 80 & 298 & 26.85 & \\
\hline & 3 & 83 & 389 & 21.34 & \\
\hline & 4 & 112 & 322 & 34.78 & \\
\hline & 5 & 80 & 369 & 21.68 & \\
\hline & 6 & 70 & 359 & 19.50 & $23.44 \pm 4.46$ \\
\hline & 7 & 60 & 345 & 17.39 & \\
\hline & 8 & 89 & 387 & 23.00 & \\
\hline & 9 & 82 & 338 & 24.26 & \\
\hline & 10 & 65 & 326 & 19.94 & \\
\hline & 11 & 83 & 356 & 23.31 & \\
\hline & 12 & 86 & 334 & 25.75 & \\
\hline
\end{tabular}

** $\mathrm{P}<0.01$ represents very significant differences related to fertile controls. SOD, superoxide dismutase. SOD, superoxide dismutase; DFI, DNA fragmentation index. 